\title{
MODELLING AND ANALYSIS OF MULTICOPTER FRAME AND PROPELLER
}

\author{
GopichandAllaka $^{1}$, B.D.P.P.S.L.Anasuya ${ }^{2}$, Ch.Yamini ${ }^{3}$, N.N.Vaidehi ${ }^{4}$, Y. Venkata Ramana $^{5}$ \\ ${ }^{1}$ Associate Professor, ${ }^{2,3,4,5}$ Student, Dept of Mechanical Engg, Swarnandhra College of Engineering \& Technology, AP
}

\begin{abstract}
Man with the advancement of technology invented various UAV's and flying machines. These can be used for various purposes. Most of them are extensively used for defense purposes and the rest are used even in domestic purposes. A Quadrocopter, commonly called as Quadrotor, is a UAV (Unmanned Aerial Vehicle) which was propelled and lifted by four rotors. Battery was used as a power source to run the motors, thereby propellers.

Modeling and Analyzing are the two important unavoidable steps in manufacturing any component or structure. In this paper we were concentrating on design and analysis of a Quadrocopter frame and propeller. The modeling was carried out using advanced software, named PRO-E while the analysis of components was done using ANSYS 13.0 version. The results obtained were compared with the various properties of frame materials which tell us whether the design made was safe or not.
\end{abstract}

Keywords: Ansys, Multicopter, Pro/E

$* * *$

\section{INTRODUCTION}

\subsection{Introduction to multicopter}

A multirotor or multicopter is a rotorcraft with more than two motors. Multirotors often use fixed-pitch blades, whose rotor pitch does not vary as the blades rotate; control of vehicle motion is achieved by varying the relative speed of each rotor to change the thrust and torque produced by each .Multicopters are the unmanned air vehicles mainly used for defense purposes. Apart from defense there are several other uses of multicopters. The applications of multicopters are: Aerial Photography, Crop dusting, Disaster Response, Accident investigation

As advancement a multicopter can also be used for the transportation of persons.

\subsection{Quadrocopter}

The basicdynamical model of the quadcopter is the starting point for all of the studies but more complex aerodynamic properties has been introduced by Hoffman and Haung [1, 2]. A quad rotor, also called a quad rotor helicopter or quad copter, is a multicopter that is lifted and propelled by four rotors.

Quad rotors are classified as rotor copter, as opposed to fixedwing aircraft, because their lift is generated by a set of revolving narrow-chord airfoils. Unlike most helicopters, quad rotors generally use symmetrically pitched blades; these can be adjusted as a group, a property known as 'collective', but not individually based upon the blade's position in the rotor disc, which is called 'cyclic' (see helicopter). Control of vehicle motion is achieved by altering the pitch and/or rotation rate of one or more rotor discs, thereby changing its torque load and thrust/lift characteristics.

\section{ABOUT PRO-E}

Pro/ENGINEER is a computer graphics system for modelling various mechanical designs and for performing related design and manufacturing operations. The system uses a 3D solid modelling system as the core, and applies the feature-based, parametric modelling method.

Pro/ENGINEER is a feature-based, parametric solid modelling system with many extended design and manufacturing applications. Assembly, processing, manufacturing and other disciplines are using the unique characteristics of these areas. To these features by setting parameters (including not only geometry, but also non-geometric properties), and then modify the parameters are easy to design iterations many times, to achieve product development.

\section{ABOUT ANSYS}

ANSYS, Inc. is an engineering simulation software (computer-aided engineering, or CAE) developer that is headquartered south of Pittsburgh in Canonsburg, Pennsylvania, United States. ANSYS offers a comprehensive range of engineering simulation solution sets providing access to virtually any field of engineering simulation that a design process requires. The tools in ANSYS put a virtual product 
through a rigorous testing procedure such as crashing a car into a brick wall before it becomes a physical object.

The ANSYS Workbench platform is the framework upon which the industry's broadest and deepest suite of advanced engineering simulation technology is built. With bi-directional CAD connectivity, powerful highly-automated meshing, project-level update mechanism, pervasive parameter management and integrated optimization tools, the ANSYS Workbench platform delivers unprecedented productivity, enabling Simulation Driven Product Development.

\section{PRO/E DESIGNS}

\subsection{Quadrocopter Frame}

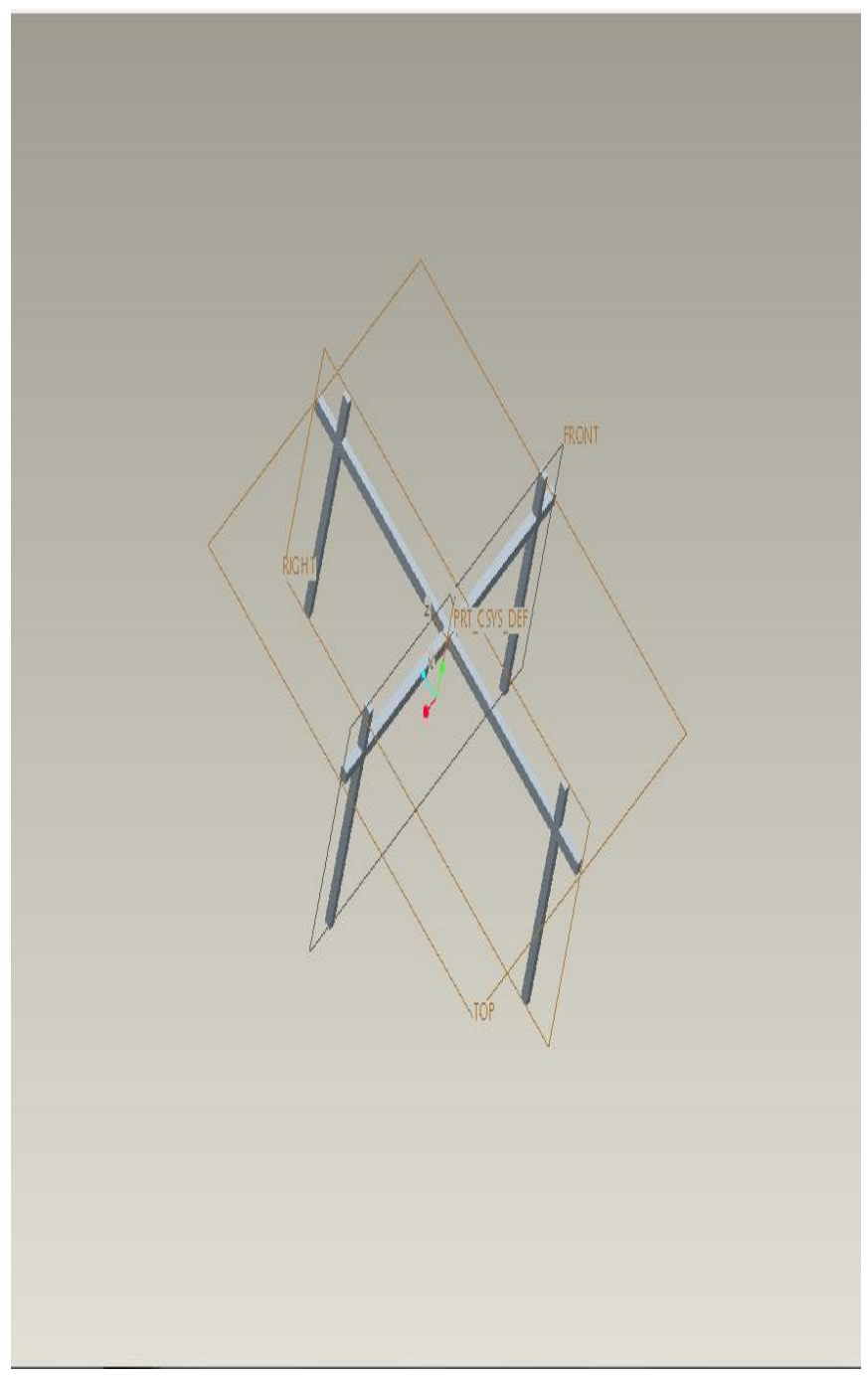

Fig:4.1 Quadrocopter Frame

\subsection{Propeller}

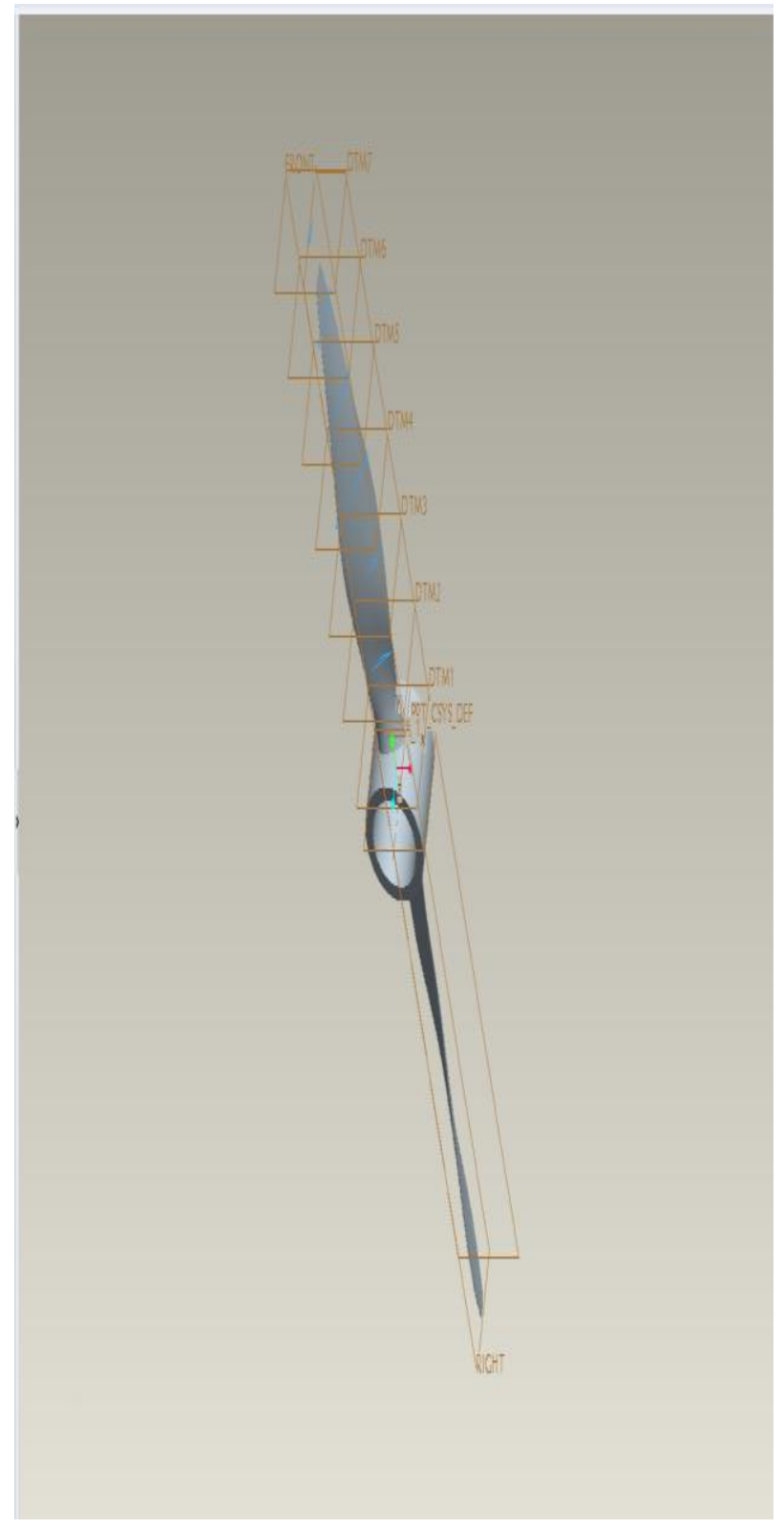

Fig: 4.1Propeller

\section{ANSYS RESULTS:}

\subsection{Analysis of Quadrocopter Frame:}

The analysis carried out is Structural Analysis. Load applied is $600 \mathrm{~N}$ i.e the approximate load for lifting a person using a quadrocopter. Applying a load of $600 \mathrm{~N}$ and performing the structural analysis we have obtained equaivalent stress which is within the limits. 


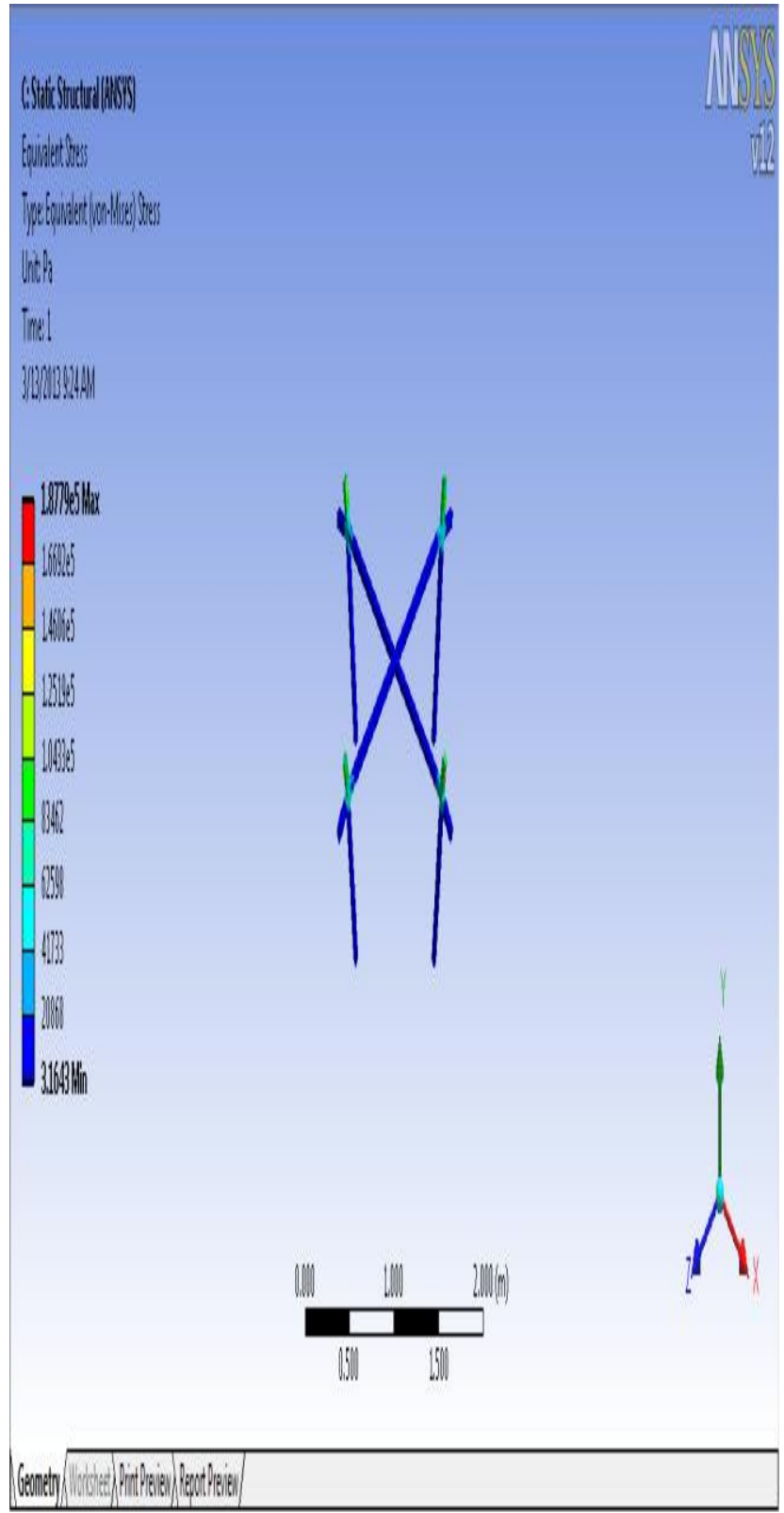

Fig 5.1: Analysis of Quadrocopter Frame

The Ultimate Tensile Stress of Structural Steel is 4.6 e+008 $\mathrm{Pa}$. The Max equivalent stress that is obtained is $1.669 \mathrm{e}+005$ $\mathrm{Pa}$. Hence the structure is safe.

\subsection{Analysis of Propeller:}

The propeller is also analysed using the structural analysis. For the analysis of Propeller it is given a rotational velocity of 9600rpm and the analysis is performed. The equivalent stress we have obtained is within the limit.

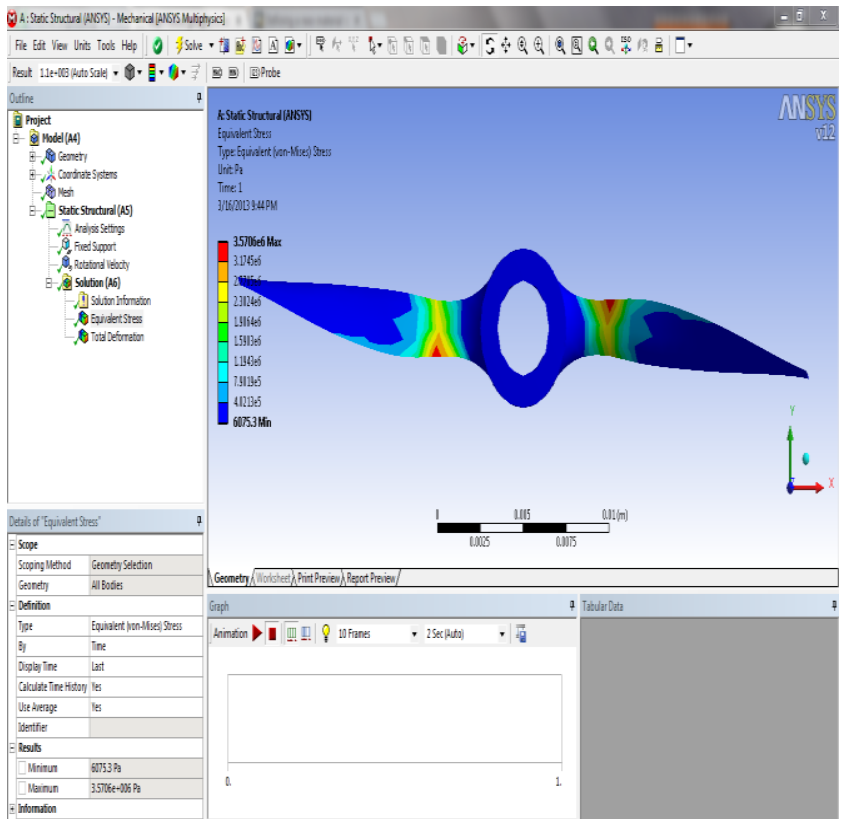

Fig5.2: Analysis of Propeller

The Ultimate Tensile Stress of Structural Steel is 4.6 e +008 $\mathrm{Pa}$. The Max equivalent stress that is obtained is $3.75 \mathrm{e}+006$ $\mathrm{Pa}$. Hence the structure is safe

\section{CONCLUSIONS}

The propeller is designed using the airfoil section S7075. By importing the airfoil section onto the datum planes design is carried out. Analysis of the Propeller and Frame are carried out using the Ansys workbench software and the results we have obtained are within the limits. AnsysWorkBench is the most efficient software for analyzing the propeller structure.

\section{REFERENCES}

[1] G. M. Hoffmann, H. Huang, S. L. Waslander, and C. J. Tomlin, "Quadrotor

helicopter flight dynamics and control: Theory and experiment," Proceedings

of the AIAA Guidance, Navigation and Control Conference and Exhibit, Aug.

2007.

[2] H. Huang, G. M. Hoffmann, S. L. Waslander, and C. J. Tomlin, "Aerodynamicsand control of autonomous quadrotor helicopters in aggressive maneuvering,"IEEE International Conference on Robotics and Automation, pp. 3277-3282,

May 2009.

[3]www.kkmulticopter.kr

[4]Pro/Engineer wildfire 4.0 by Sham Tickoo

[5]Ansys workbench software release 12.0 tutorial by Jack Zecher and FereydoonDadkhah. 